\title{
Pharmacological activation of the souble guanylate cyclase inhibits pressure overload-induced cardiac hypertrophy
}

\author{
Balázs Tamás Németh*, Csaba Mátyás, Attila Oláh, László Hidi, Mihály Ruppert, Árpád Lux, \\ Dalma Kellermayer, Gergõ Merkely, Béla Merkely, Tamás Radovits \\ Semmelweis University, Heart and Vascular Center, Budapest, Hungary
}

Background and Aim: Pathological cardiac hypertrophy is observed in pressure overload of the left ventricle. Elevated intracellular cGMP-levels have been reported to prevent the development of pathological myocardial hypertrophy. We investigated the effects of the chronic activation of the cGMP producing enzyme, soluble guanylate cyclase (sGC) by cinaciguat in a rat model of pressure overload-induced cardiac hypertrophy.

Methods: We performed aortic banding $(A B)$ to evoke pressure overload-induced cardiac hypertrophy in our rats. Sham operated on animals served as controls. Experimental and control groups were treated with $10 \mathrm{mg} / \mathrm{kg} / \mathrm{day}$ cinaciguat (Cin) or placebo (Co) p.o., respectively. The development of cardiac hypertrophy was investigated by echocardiography. We performed the left ventricular (LV) pressurevolume analysis with a pressure-conductance microcatheter to assess the cardiac function. In addition to our functional experiments, histological and molecular biological measurements were carried out.

\section{Received: $28^{\text {th }}$ Apr 2014}

*Address for correspondence: Semmelweis University, Heart and Vascular Center, Gaál József street 9, H-1122 Budapest, Hungary.

Phone: +36206632946

E-mail: nemethbl@gmail.com
Results: Echocardiography showed marked myocardial hypertrophy in the $\mathrm{AB}-\mathrm{Co}$ group (left ventricular mass index (LVMi): $3.15 \pm 0.09$ AB-Co vs. $2.13 \pm 0.04 \mathrm{~g} / \mathrm{kgBW}$ Sham-Co) which was verified by post mortem investigation of the hearts (heart weight/tibial length ratio $(\mathrm{HW} / \mathrm{TL})$ : $0.384 \pm 0.015$ AB-Co vs. $0.293 \pm 0.008 \mathrm{~g} / \mathrm{cm}$ Sham-Co) and by histology (cardiomyocyte diameter (CD): $17.37 \pm 0.04$ AB-Co vs. $14.55 \pm 0.12 \mu \mathrm{m}$ Sham-Co). Increased left ventricular dimensions (left ventricular end-diastolic volume: $414 \pm 19$ AB-Co vs. $341 \pm 19 \mu \mathrm{l}$ Sham-Co) were observed while the ejection fraction and fractional shortening remained unchanged. $\mathrm{Ci}-$ naciguat did not alter blood pressure $(182.27 \pm 7.86 \mathrm{AB}-\mathrm{Co}$ vs. $174.63 \pm 4.53 \mathrm{mmHg} A B-C i n, p=n . s$.), but effectively attenuated the left ventricular hypertrophy (LVMi: $2.64 \pm 0.06$ $\mathrm{g} / \mathrm{kgBW}, \mathrm{HW} / \mathrm{TL}: 0.339 \pm 0.009 \mathrm{~g} / \mathrm{cm}, \mathrm{CD}: 15.08 \pm 0.10 \mu \mathrm{m}, \mathrm{p}$ $<0.05$ vs. AB-Co).

Conclusion: Our results demonstrate that chronic stimulation of the NO-cGMP signaling pathway by pharmacological activation of the soluble guanylate cyclase might be a novel therapeutic approach in the prevention of pathological myocardial hypertrophy.

KEYWORDS: aortic banding, pressure overload, cinaciguat, myocardial hypertrophy.

CITTATION: Cardio Croat. 2014;9(5-6):245. 